\title{
Rapid Identification of the Chemical Components of Ilex rotunda Thunb Using UPLC-Q-TOF-MS/MS
}

\author{
Xinxin Chen $\mathbb{D}^{1,2}$ Junmao Li, ${ }^{3}$ Renyikun Yuan, ${ }^{1,3}$ Youqiong Zhuo, ${ }^{1,2}$ Yangling Chen, ${ }^{1,2}$ \\ Chenwei Zhang, ${ }^{1,2}$ Ming Chen, ${ }^{1,2}$ Hong-Wei Gao $\mathbb{D}^{1,},{ }^{1,2}$ Zhenjie Liu $\mathbb{D}^{1,2}$ Yulin Feng, ${ }^{3}$ \\ Zheng-Tao Wang, ${ }^{4}$ and Shilin Yang ${ }^{1,2}$ \\ ${ }^{1}$ College of Pharmacy, Guangxi University of Chinese Medicine, Nanning 530000, China \\ ${ }^{2}$ Guangxi Engineering Technology Research Center of Advantage Chinese Patent Drug and Ethnic Drug Development, \\ Nanning 530020, China \\ ${ }^{3}$ Jiangxi University of Chinese Medicine, Nanchang 330002, China \\ ${ }^{4}$ Shanghai University of Chinese Medicine, Shanghai 201203, China
}

Correspondence should be addressed to Hong-Wei Gao; gaohongwei06@126.com and Zhenjie Liu; rainman982@126.com

Received 29 May 2021; Revised 19 August 2021; Accepted 8 September 2021; Published 30 September 2021

Academic Editor: Yun Wei

Copyright (C) 2021 Xinxin Chen et al. This is an open access article distributed under the Creative Commons Attribution License, which permits unrestricted use, distribution, and reproduction in any medium, provided the original work is properly cited.

Ilicis Rotundae Cortex (IRC) consists of the bark of Ilex rotunda Thunb, and its chemical constituents mainly include flavonoid glycosides, phenols, and triterpenoid saponins. In this study, a preliminary analysis was performed to identify and obtain the chemical components from IRC to better control the quality of the medicinal materials and provide a chemical basis for the study of the efficacy of the active components. Simple and efficient sample pretreatment and ultrasonic-assisted extraction methods were used to analyze the mass spectrum fragments and fracture modes in the anion mode by UPLC-Q-TOF-MS/MS. Using a two-step strategy, the neutral loss, diagnostic ions, and characteristic fragments were studied to screen diverse skeletons and substitutions, and the possible compounds were identified by comparison with databases. The representative compounds were compared with the standard, and the mass spectrogram was found to match perfectly. Thus, our findings reveal that this method is feasible and reliable and can be used to analyze the chemical components of IRC. We identified 105 compounds, including 22 triterpenoid saponins, 15 chlorogenic acids, 33 phenylpropanoids and phenylpropanosides, 3 iridoids, 1 flavonoid, 10 lignans, 12 glycosides, and 9 other compounds. This method lays the foundation for further elucidating the pharmacodynamics of IRC and provides a practical method for the identification of IRC.

\section{Introduction}

Ilicis Rotundae Cortex (IRC) is a special medicinal material used by the Yao nationality in China and has been included in the Chinese Pharmacopoeia 2020 Edition. Modern pharmacological studies have shown that IRC extract has several effects such as reducing coronary artery flow, slowing heart rate, improving hypoxia tolerance, and antiarrhythmic effects. Notwithstanding that it is often used in the treatment of cardiovascular diseases in a clinical setting, the active components and mechanism of action of IRC remain unclear [1-3].

Scholars both overseas and in China have extensively studied the chemical composition, pharmacological actions, and clinical applications of this drug [4-8]. The main constituents are triterpenes and their saponins, sesquiterpenes, diterpenes, flavonoid glycosides, aromatic compounds, and other chemical components, among which triterpenes and their saponins are one of the main chemical components. Although some progress has been made in the pharmacological study of IRC, research on its chemical components is still not comprehensive. Thus, it is urgent to lucubrate these rich medicinal material resources to provide a chemical component basis for the further later study of medicinal materials.

Nowadays, liquid mass spectrometry (MS) has been widely used for the qualitative and quantitative analyses of 
complex samples. Quadrupole time-of-flight (Q-TOF) MS can be used to obtain both precise mass numbers of the MS and MS/MS for small molecular compounds and proteins [9-12]. However, in the case of complex samples, characterization of active compounds is still a challenge owing to the large and complex MS data. Several techniques, such as neutral loss and diagnostic ion filtration, have been developed to eliminate or reduce interfering ions and to achieve the goal of high-speed identification and screening of active compounds.

In this study, we used neutral loss, diagnostic ion screening, and characteristic ion fragmentation as filtering strategies assisted by database matching and compared our findings with the standard. Lastly, a method for the rapid identification of the chemical constituents of IRC extract was achieved.

\section{Experimental}

2.1. Chemicals and Reagents. IRC was acquired from Guangxi Yulin Zhen Caotang Chinese Medicinal Decoction Pieces Co., Ltd. (Guangxi Zhuang Autonomous Region, China). Acetonitrile and methanol (Optima LC/MS, suitable for UHPLC-UV) were obtained from Fisher (New Jersey, USA). Formic acid was purchased from Aladdin (Bay City, USA). Distilled water was purchased from Hangzhou Wahaha Group Co., Ltd. (Hangzhou, China). The fifteen standard compounds of IRC, including 1,4-dicaffeoylquinic acid, 3,4-dicaffeoylquinic acid, 3,5-dicaffeoylquinic acid, chlorogenic acid, neochlorogenic acid, cryptochlorogenic acid, syringin, kudinoside $\mathrm{H}$, ilexgenin A, 3,4-dihydroxybenzoic acid, 3,4-dihydroxybenzaldehyde, rotundic acid, caffeic acid, ilexsaponin A, and pedunculoside, were purchased from Chengdu Must Bio-Technology Co., Ltd. (Chengdu, China). All medicinal materials were identified by experts, and standard compounds were analyzed using HPLC to confirm purity $>98 \%$.

2.2. Sample Preparation. IRC was crushed, and about $1.0 \mathrm{~g}$ was weighed, placed in a conical flask, and extracted using $40 \mathrm{~mL}$ of $70 \%$ methanol ( $\mathrm{v} / \mathrm{v})$ for $2 \mathrm{~h}$ by ultrasound. The supernatant was centrifuged at $12000 \mathrm{rpm}$ for $15 \mathrm{~min}$ and stored at $4^{\circ} \mathrm{C}$ for UPLC-Q-TOF-MS/MS analysis [13-16].

All standard compounds were accurately weighed $(1.0 \mathrm{mg})$, dissolved in $1.0 \mathrm{~mL}$ methanol, diluted to ppb level, mixed at $50 \mu \mathrm{L}$ each, and stored at $4^{\circ} \mathrm{C}$ for UPLC-Q-TOFMS/MS analysis.

2.3. Instrumentation Conditions. A Nexera X2 LC-30A ultrahigh-performance liquid chromatograph (Shimazu, Japan) series Q-TOFTM 5600 high-resolution mass spectrometer equipped with an ESI ion source was used.

2.3.1. UPLC Conditions. A Welch $\mathrm{C}_{18}$ column $(2.1 \mathrm{~mm} \times$ $100 \mathrm{~mm}, 1.7 \mu \mathrm{m}$ ) was used at an optimal temperature of $40^{\circ} \mathrm{C}$ and a detection wavelength of $250 \mathrm{~nm}$. The flow rate and sample injection volume were rigidly controlled at $0.3 \mathrm{~mL} /$ min and $1 \mu \mathrm{L}$, respectively. The mobile phase was $0.1 \%$ aqueous formic acid (A) and acetonitrile (B). The elution gradient was as follows: $0.01 \mathrm{~min}, 5 \% \mathrm{~B} ; 1 \mathrm{~min}, 5 \% \mathrm{~B} ; 4 \mathrm{~min}$, $10 \% \mathrm{~B} ; 10 \mathrm{~min}, 15 \% \mathrm{~B} ; 20 \mathrm{~min}, 16 \% \mathrm{~B} ; 27 \mathrm{~min}, 31 \% \mathrm{~B} ; 33 \mathrm{~min}$, $59 \%$ B; 41 min, 95\% B; 42 min, 95\% B; and 42.1 min, Stop B.

2.3.2. Mass Spectrometry Conditions. An electrospray ion source (ESI) was used, and scanning was in the negative ion mode over a scanning range of $\mathrm{m} / z 5-1250$. The MS parameters were optimized as follows: ion source temperature (TEM), $550^{\circ} \mathrm{C}$; ion spray voltage floating (ISVF), $-4.5 \mathrm{kV}$; curtain gas (CUR), $35 \mathrm{kPa}$; atomization gas (GS1) and auxiliary gas (GS2), $55 \mathrm{kPa}$; and declustering potential (DP), $-100 \mathrm{~V}$ [17-19]. TOF-MS-IDA-MS/MS was used for data acquisition. The TOF/MS first-level prescan was $150 \mathrm{~ms}$, the ion accumulation time of triggering the second-level scan TOF/MS/MS was $100 \mathrm{~ms}$, the collision energy (CE) was $-35 \mathrm{eV}$, and the collision energy superposition (CES) was $25 \mathrm{~V}$. The methods to trigger the second level were information-dependent acquisition (IDA), multiple quality deficit (MMDF), and dynamic background subtraction (DBS), which were the superior and the most appropriate parameters to trigger the second-level scan. The second-level scan was a priority for samples that met the conditions.

2.4. Data Processing. Analyst TF 1.6 and XIC Manager of PeakView (AB Company in the US) were used for data processing.

\section{Results and Discussion}

3.1. Optimization of LC-MS/MS Conditions. IRC is a complex system containing over a hundred compounds. To obtain high-quality MS peaks, an appropriate extraction method, mobile phase, and elution rate must be selected to improve the reproducibility, selectivity, and peak shape. To obtain more fragment ions for IRC identification, the positive and negative ions were compared. The sensitivity of most substances in the negative ion mode is higher than that in the positive ion mode. Furthermore, between ultrasonic extraction and thermal reflux extraction, the former was obviously superior to the latter for IRC, as reflux extraction might destroy the thermally unstable compounds. After adopting ultrasonic extraction at length, the chemical composition of IRC was analyzed in the negative ion mode. The chemical components in IRC were analyzed using a twopart strategy. As for the mobile phase, we studied the suitability of acetonitrile, methanol, pure water, and $0.1 \%$ formic acid in water and finally used acetonitrile and $0.1 \%$ formic acid in water as the mobile phases at a flow rate of $0.3 \mathrm{~mL} / \mathrm{min}$.

First, we determined the precise molecular weight of the compounds using high-resolution MS and deduced their molecular formula using MS. Next, we analyzed and classified compounds based on neutral loss, diagnostic ions, and characteristic fragments. Lastly, the typical compounds in each category were selected and compared with the standard compounds (Figure 1). The final results showed that the 


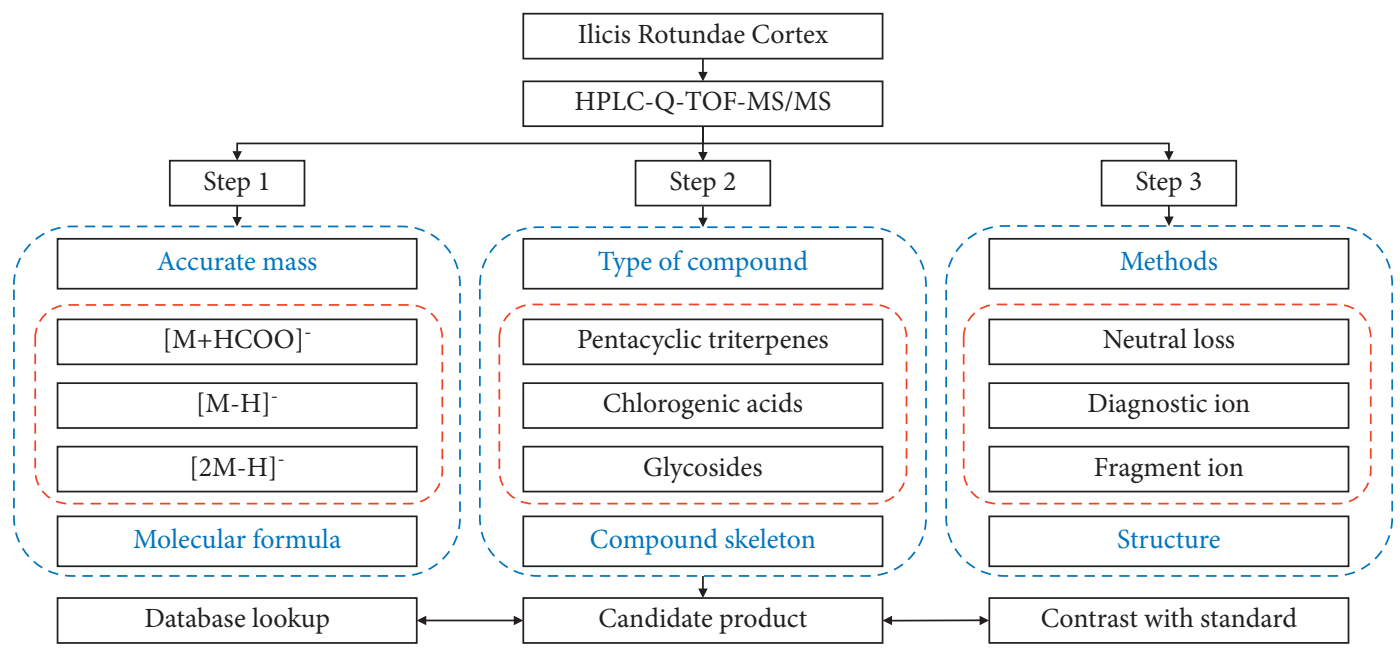

FIGURE 1: Schematic diagram of the integrative strategy.

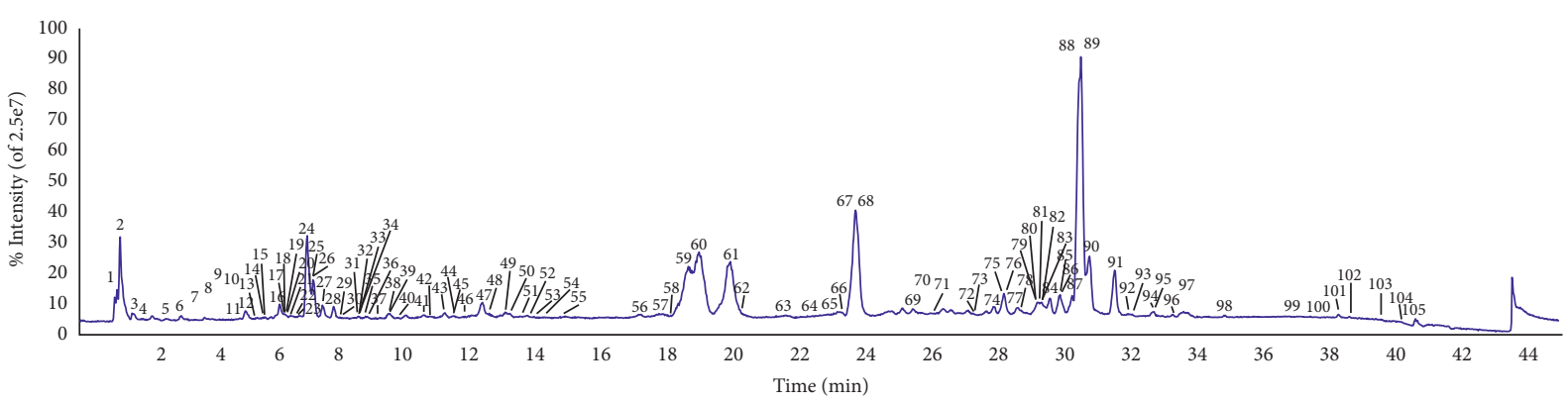

(a)

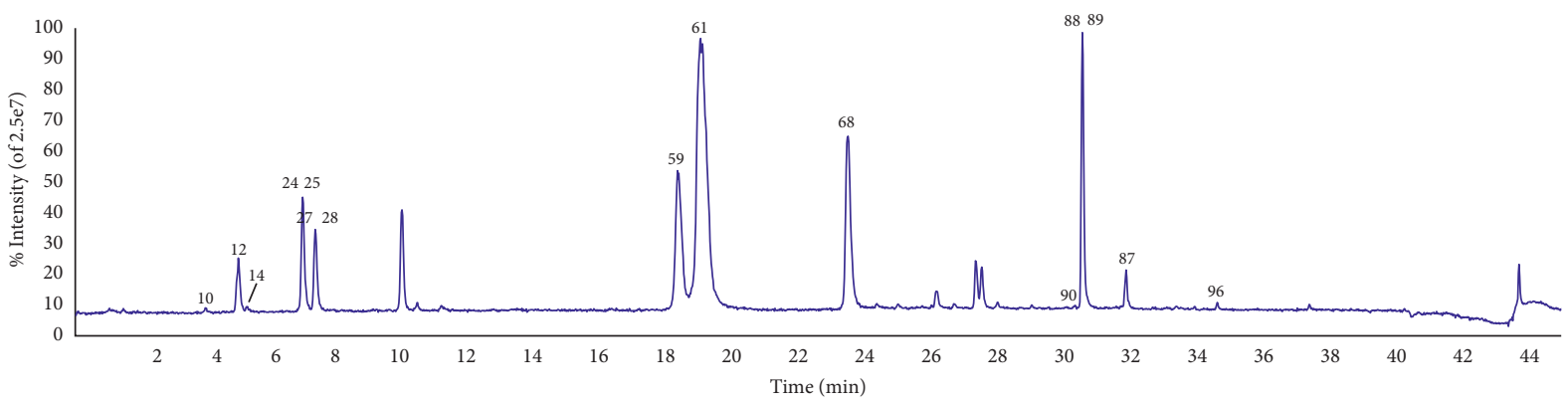

(b)

Figure 2: TIC of (a) Ilicis Rotundae Cortex (IRC) and (b) the 15 mixed standard compounds in the negative ion mode.

predicted components were completely consistent with the mass spectrum of the standard components, suggesting that our method was feasible and reliable for the prediction of chemical components in IRC.

The retention time and mass spectrum of each chemical component in IRC were analyzed. Through consulting and referring to the relevant literature and database search, the compounds were confirmed by comparing the base peak diagram with the reference material. Gratifyingly, 105 compounds in IRC were preliminarily identified, which included 22 triterpenoid saponins, 15 chlorogenic acids, 33 phenylpropanoids and phenylpropanosides, 3 iridoids, 1 flavonoid, 10 lignans, 12 glycosides, and 9 other compounds. The total ion chromatogram (TIC) for the 105 identified compounds is shown in Figure 2(a) and was sequenced using peak time. The 15 reference compounds (M10: 3,4-dihydroxybenzoic acid; M12: chlorogenic acid; M14: 3,4-dihydroxybenzaldehyde; M24: neochlorogenic acid; M25: syringin; M27: cryptochlorogenic acid; M28: caffeic acid; M59: 1,4-dicaffeoylquinic acid; M61: 3,4-dicaffeoylquinic acid; M68: 3,5-dicaffeoylquinic acid; M87: ilexsaponin A; M88: pedunculoside; M89: rotundic acid; M90: kudinoside H; M96: ilexgenin A) matched perfectly with the mass spectrum fragments and retention time of the identified components. Our findings indicated the accuracy and reliability of our method. All compounds are listed in Table 1. 


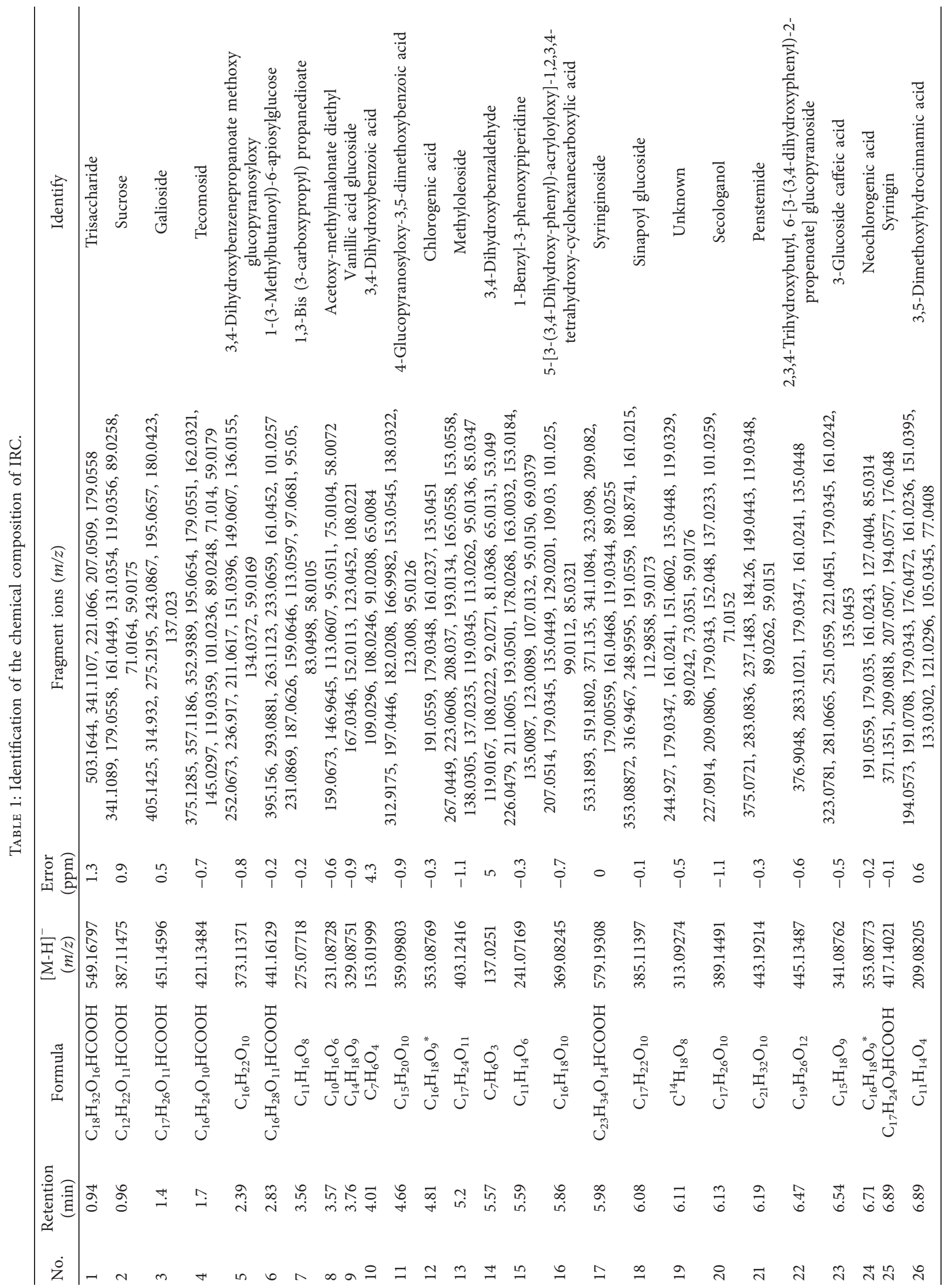




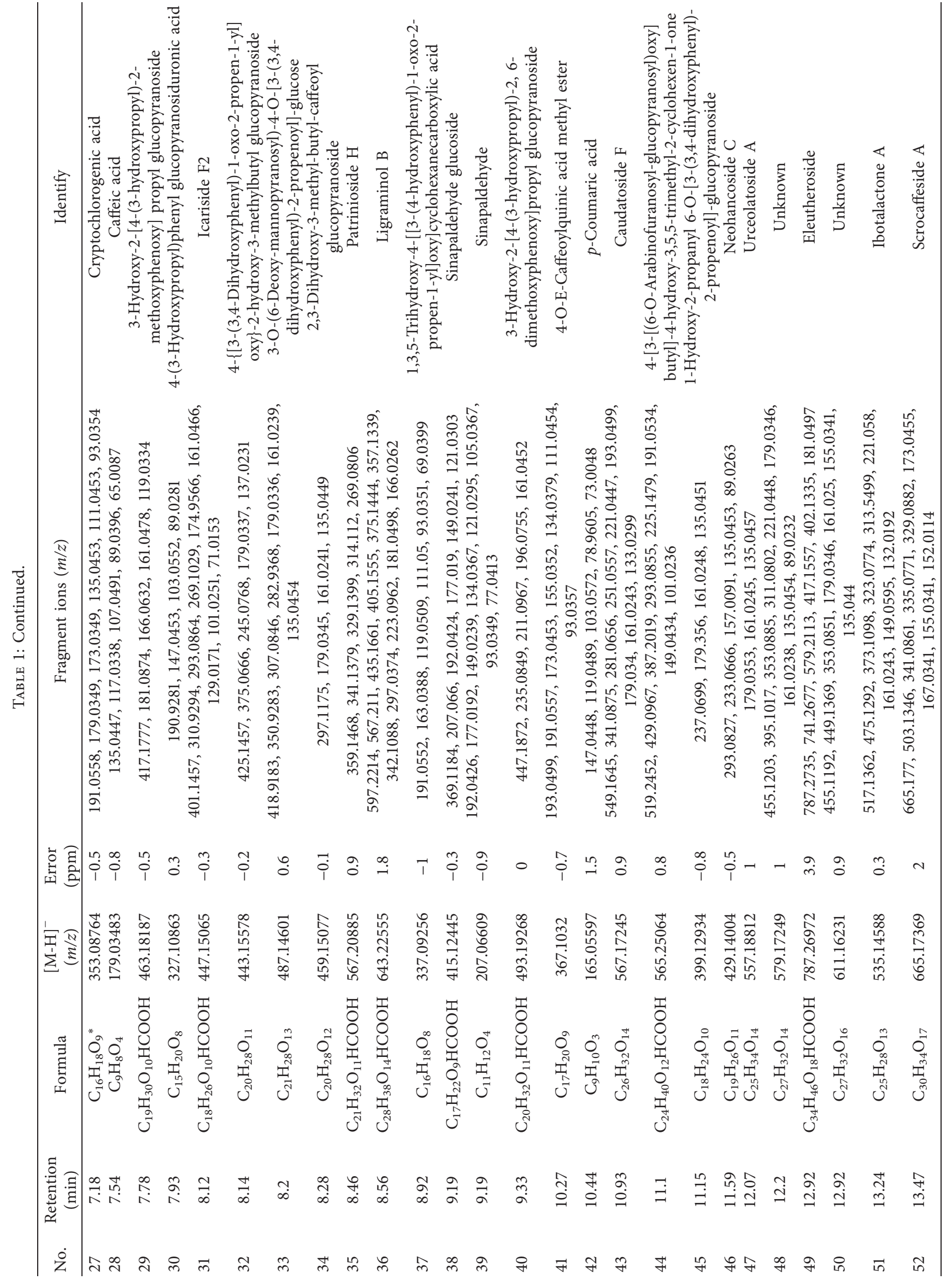




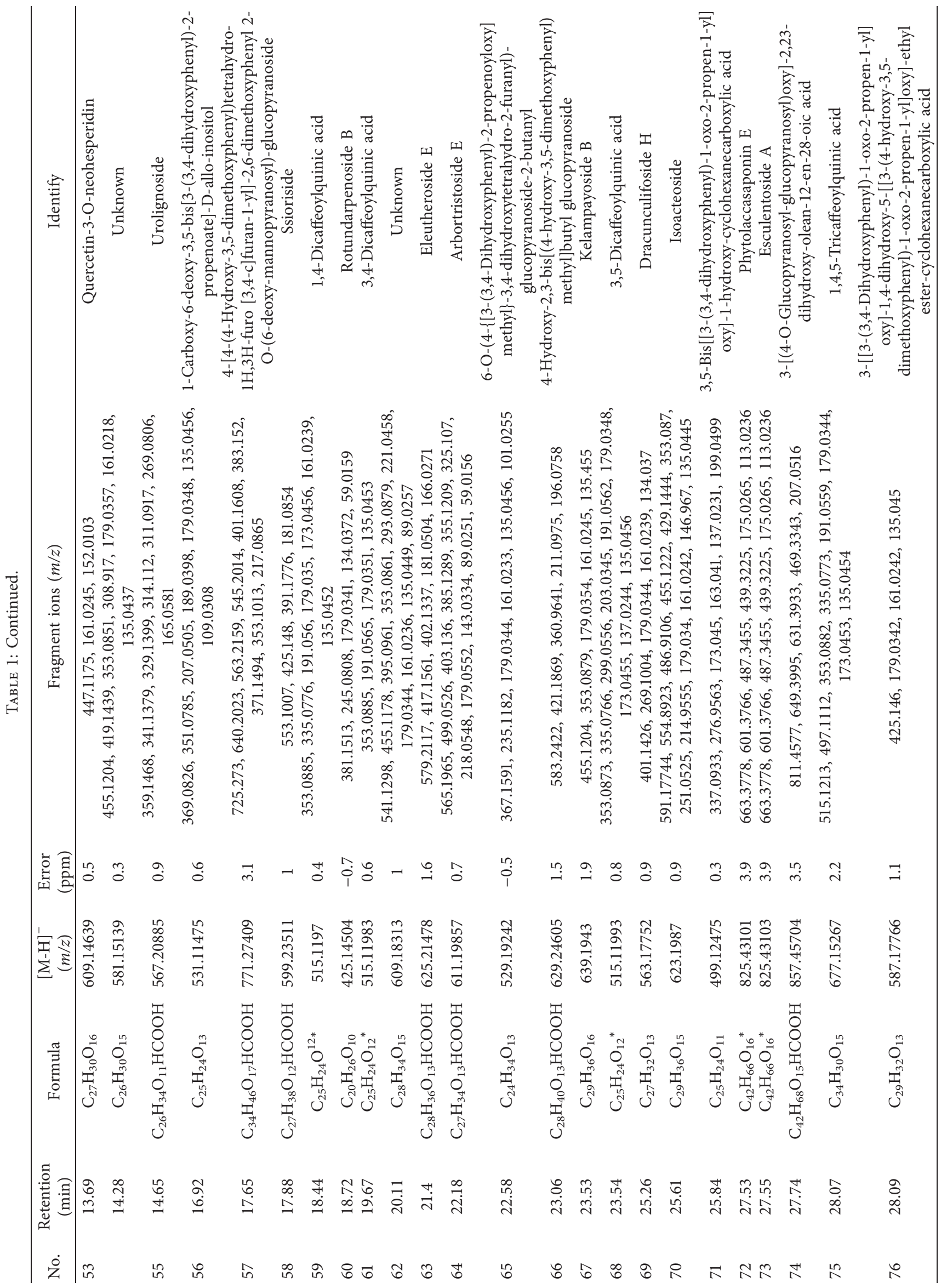




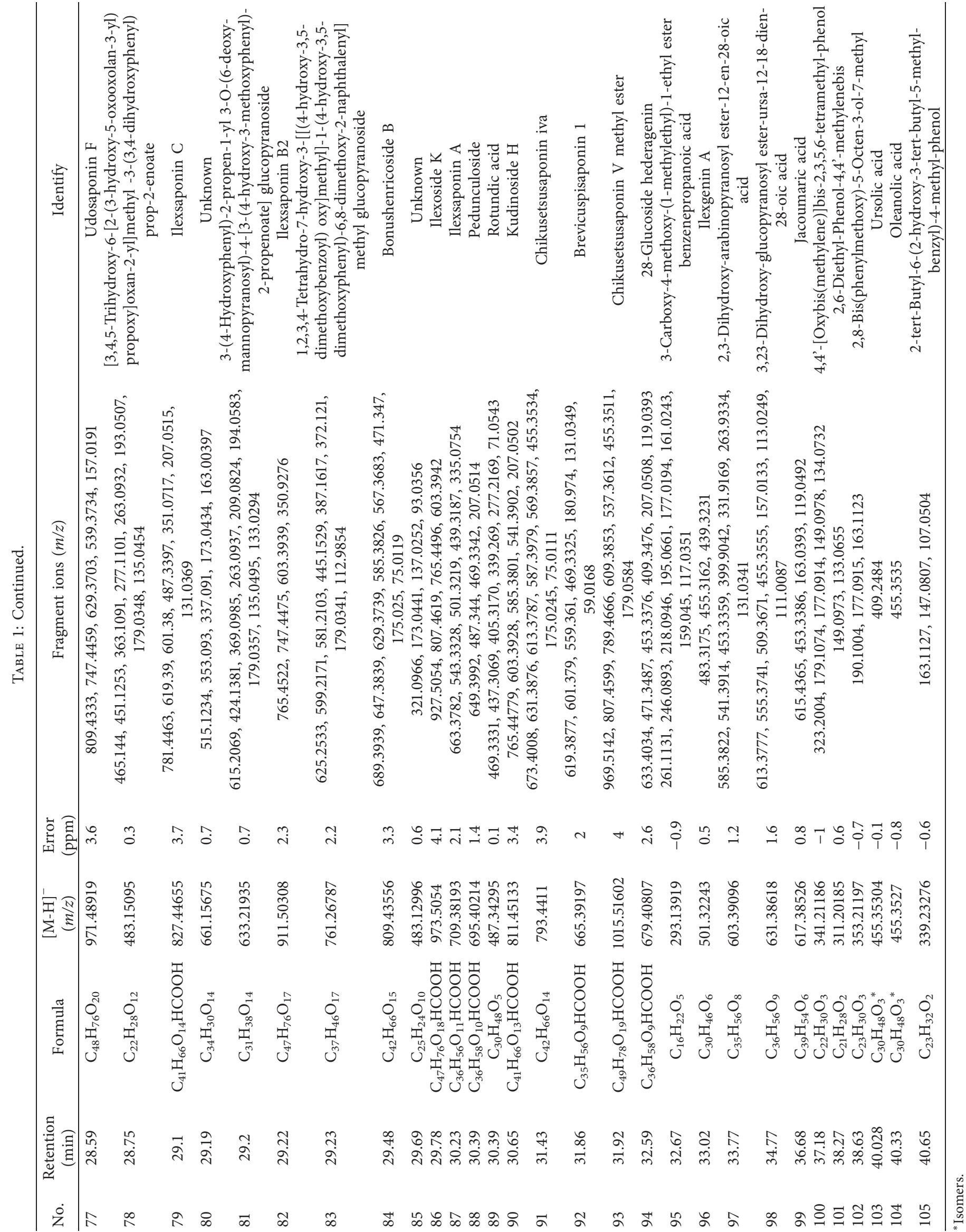




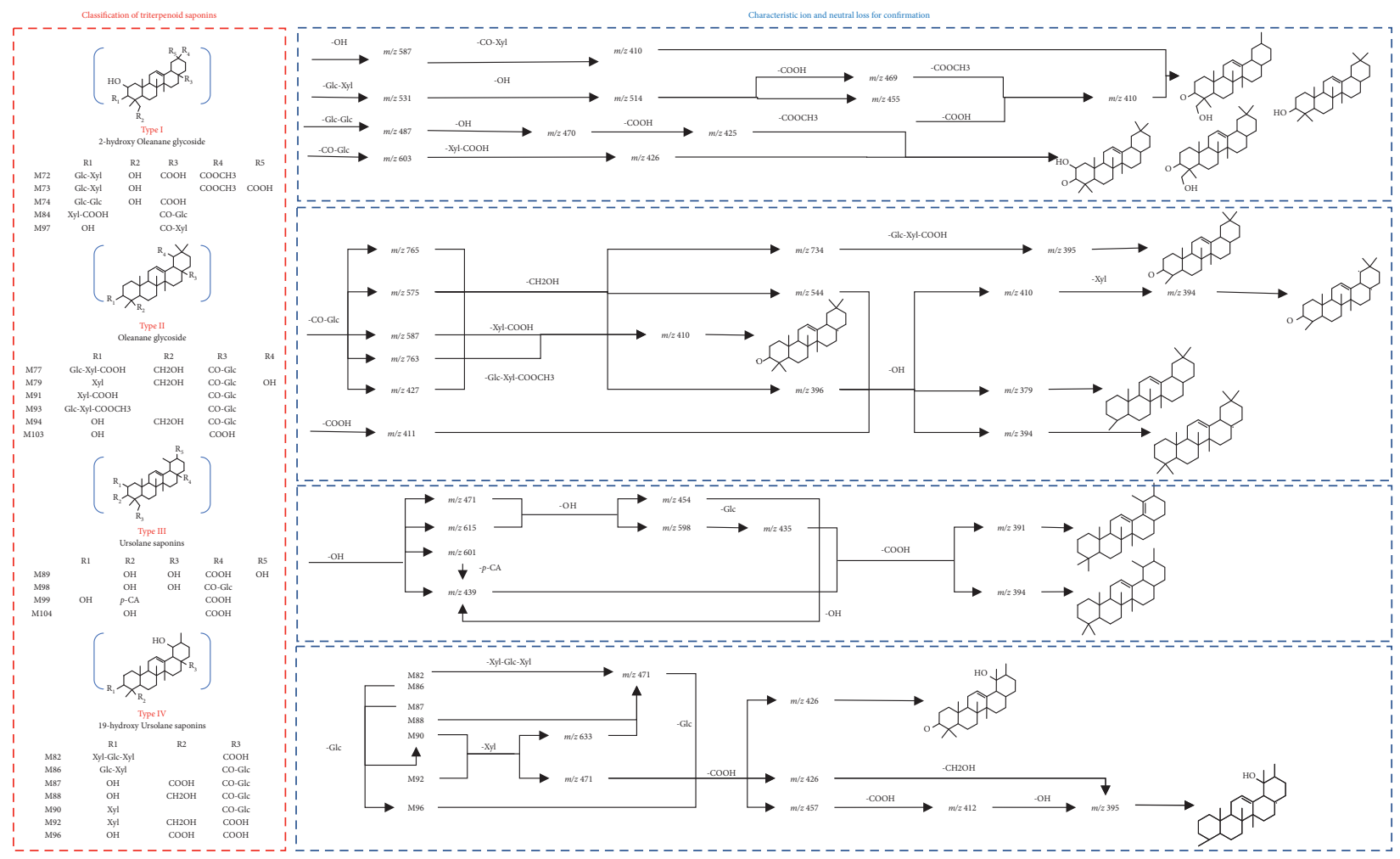

FIGURE 3: Types of triterpenes and their cracking regularity in ICR.

3.2. Cleavage Rules of Pentacyclic Triterpenes and Their Saponins. A previous study reported that IRC contains several triterpenoid saponins, mainly including the oleanane and ursolane types [20,21]. Without ring-opening, these compounds have diagnostic ions $\mathrm{m} / z$ 394, $\mathrm{m} / \mathrm{z} 410$, and $\mathrm{m} / \mathrm{z}$ 426. Confirmation is obtained between types I and IV. Compound classification, diagnostic ions, and cleavage are shown in Figure 3. In this study, 22 triterpenes and their saponins were preliminarily identified [22-24]. Conclusively, five representative standards including ilexsaponin A, pedunculoside, rotundic acid, kudinoside $\mathrm{H}$, and ilexgenin $\mathrm{A}$ were compared and confirmed.

These 22 compounds were derived from the two elementary structures of oleanolate and ursolane and classified into the following four major groups: oleanolate $(\mathrm{m} / \mathrm{z}$ 438.4226), 2-hydroxy oleanolate $(\mathrm{m} / z$ 454.4175), ursolane $(\mathrm{m} / z$ 452.4382), and 19-hydroxy ursolane $(\mathrm{m} / z$ 440.4018). After analyzing the characteristic ions in IRC based on MS, we found that $m / z 175.0265,207.0516,409.3476,439.3225$, $455.3511,469.3343,585.3826,603.3939$, and 765.4496 are the diagnostic fragments of triterpenoid saponins in IRC. The glycosidic bonds were easily broken and often produced desaccharified fragment peaks, including glucose (Glc) $\mathrm{m} / \mathrm{z}$ 162.0528 or 179.0556 , rhamnose (Rha) $m / z 146.0579$ or 163.0606, and xylose (Xyl) $\mathrm{m} / z 132.0423$ or 149.0450 . Moreover, dehydration and decarboxylation reactions were observed, which mainly occurred at the C-3, C-23, C-28, C-19, and C-30 positions.

By comparing with the control, M87 (ilexsaponin A) obtained through [M-HCOOH-Glc- $\left.\mathrm{H}_{2} \mathrm{O}-\mathrm{CO}_{2}\right]^{-}$produced $\mathrm{m} / \mathrm{z}$ 663.3797, 501.2331, 483.3147, and 439.3311
(Figure 4(a)). M88 (pedunculoside) obtained through [M$\left.\mathrm{HCOOH}-\mathrm{Glc}-\mathrm{H}_{2} \mathrm{O}-\mathrm{CH}_{3} \mathrm{OH}\right]^{-}$produced $\mathrm{m} / \mathrm{z}$ 649.4009, 487.3458, 469.3340, and 437.3066 (Figure 4(b)). M89 (rotundic acid) obtained through $\left[\mathrm{M}-\mathrm{H}-\mathrm{H}_{2} \mathrm{O}-\mathrm{CH}_{3} \mathrm{OH}-\right.$ $\left.\mathrm{CH}_{3} \mathrm{OH}\right]^{-}$produced $\mathrm{m} / z$ 487.3439, 469.3331, 437.3069, and 405.3170 (Figure 4(c)). M90 (kudinoside H) obtained through $\left[\mathrm{M}-\mathrm{H}-\mathrm{Glc}-(\mathrm{Xyl})-\mathrm{H}_{2} \mathrm{O}-\mathrm{COOH}\right]^{-}$produced $\mathrm{m} / \mathrm{z}$ 765.4479, 603.3928, 585.3801, 541.3902, 471.3500, and 453.3384 (Figure 4(d)). M96 (ilexgenin A) obtained through $\left[\mathrm{M}-\mathrm{H}-\mathrm{H}_{2} \mathrm{O}-\mathrm{HCOOH}-\mathrm{O}\right]^{-}$produced $\mathrm{m} / z$ 501.3249, 483.3175, and 439.3231 (Figure 4(e)). All fragments in the compound matched the corresponding reference materials exactly. Therefore, we could infer other compounds of the same type.

3.3. Cleavage Rules of Chlorogenic Acids. Chlorogenic acids are derived from quinic acid (QA) and were distinguished according to the number and types of substituents [25]. The diagnostic ions at $\mathrm{m} / z 191.0556$ and 173.045 corresponded to $[\mathrm{QA}-\mathrm{H}]^{-}$and $\left[\mathrm{QA}-\mathrm{H}-\mathrm{H}_{2} \mathrm{O}\right]^{-}$. However, it was not possible to ascertain the precise substitution sites using UPLC-Q-TOF-MS/MS. Likewise, we confirmed our finding by comparing them with the literature or standard compounds. A total of 15 chlorogenic acids were identified in this study, and their structures are presented in Figure 5. Eleven types were identified based on their substituents, as well as their positions and the number. Cracking is mainly achieved by removing fragments such as QA, SA, CA, and $p$-CA. QA has $m / z 192.0634$, and the fragment [QA-H] ${ }^{-}$has $m / z$ 191.0561. Similarly, [SA-H] 
A

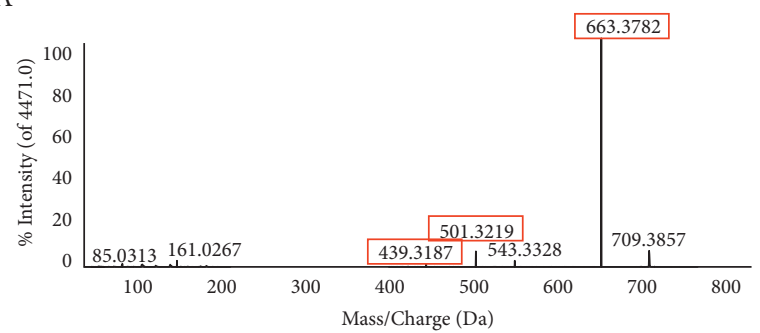

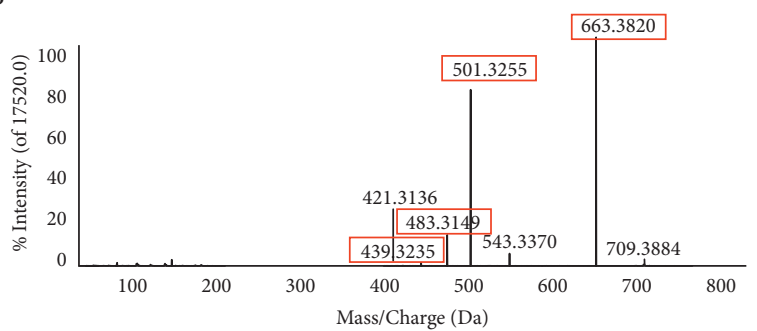

C

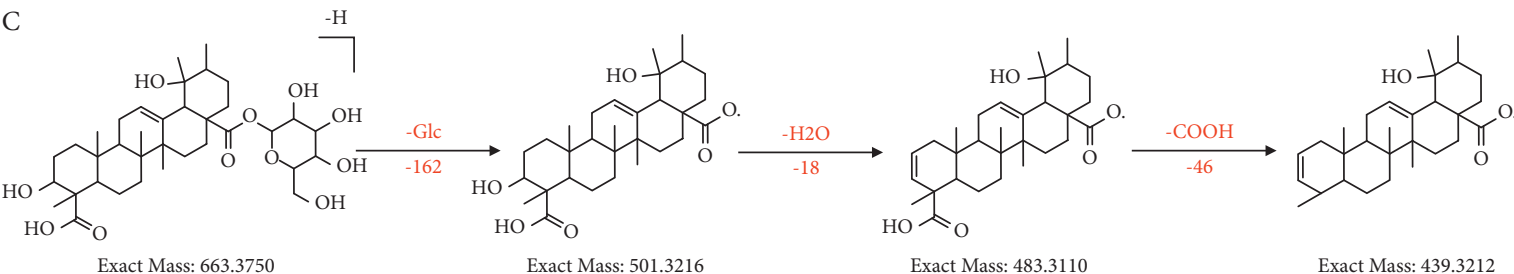

(a)

A

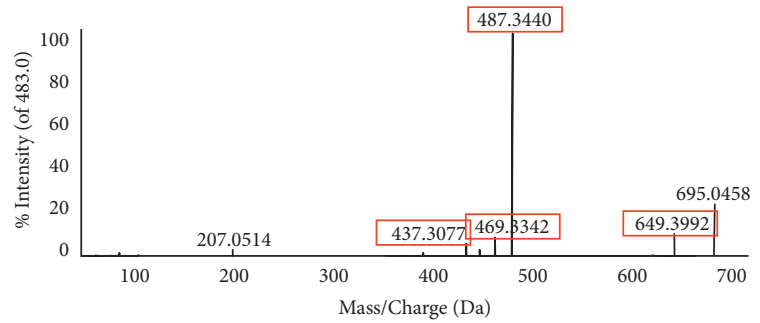

B

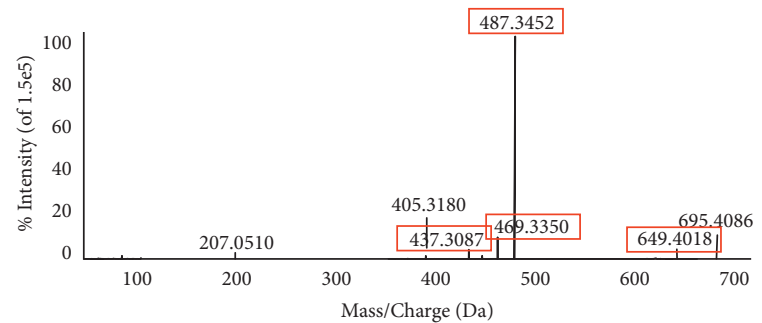



Exact Mass: 649.3957

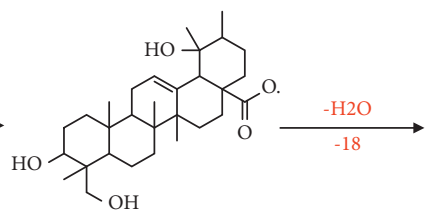

Exact Mass: 487.3423

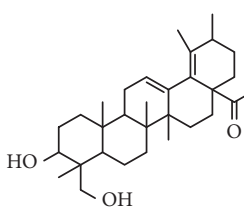

Exact Mass: 469.3318

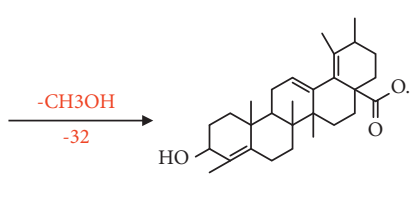

Exact Mass: 437.3056

(b)

FIgUre 4: Continued. 
A

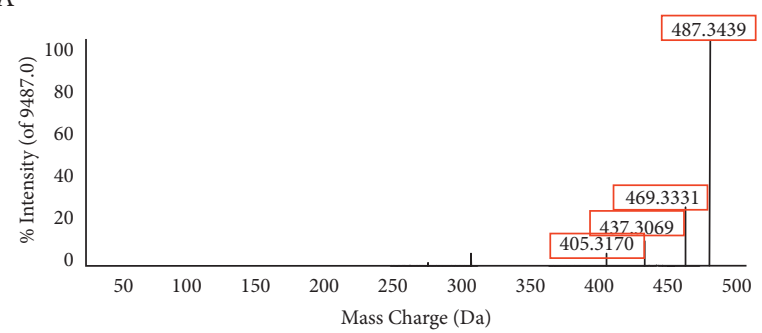

C

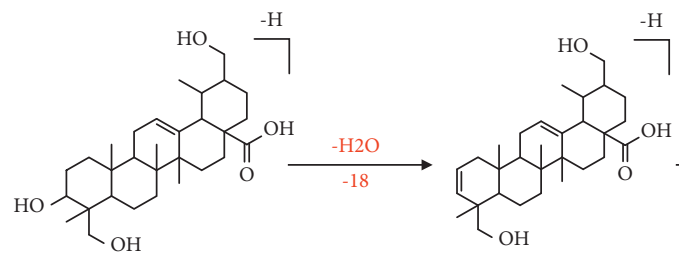

Exact Mass: 487.3429
$\mathrm{OH}$
Exact Mass: 469.3323
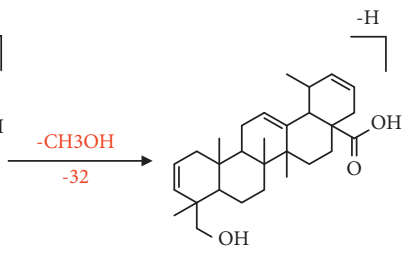

Exact Mass: 437.3061

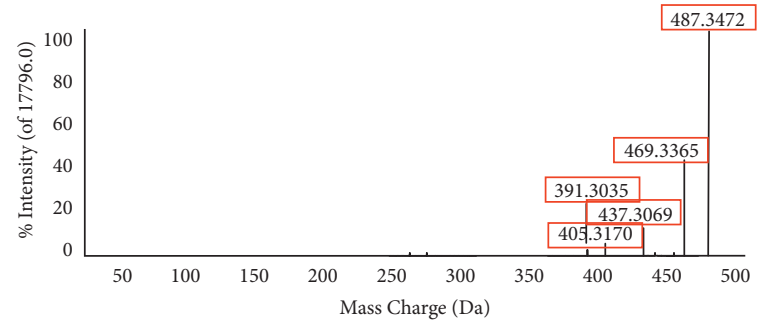

(c)

A

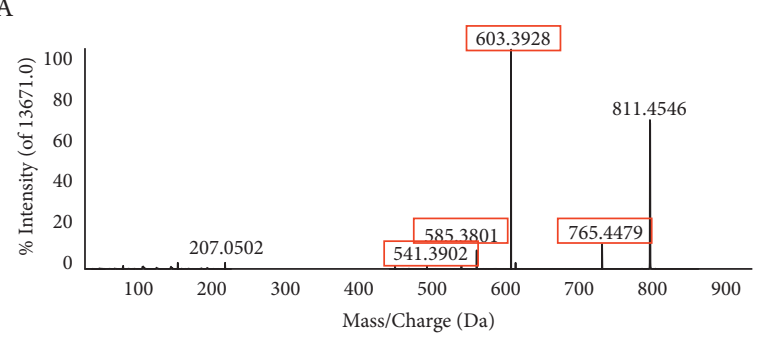

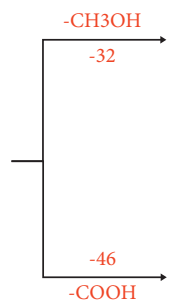

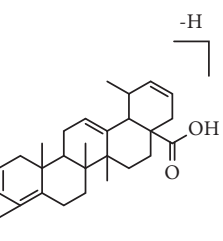

Exact Mass: 405.2799

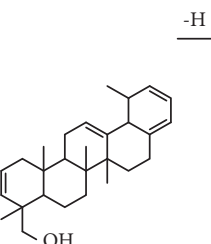

Exact Mass: 391.3006

B

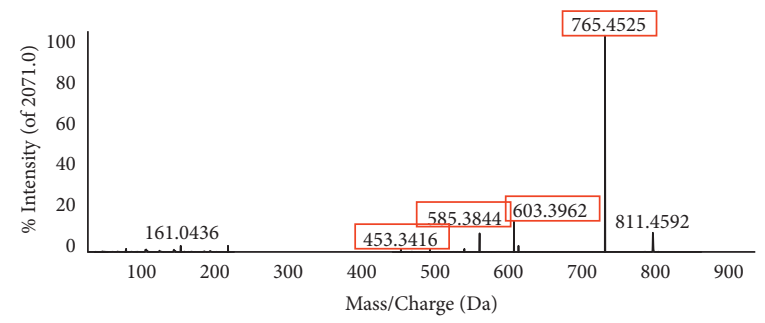

C

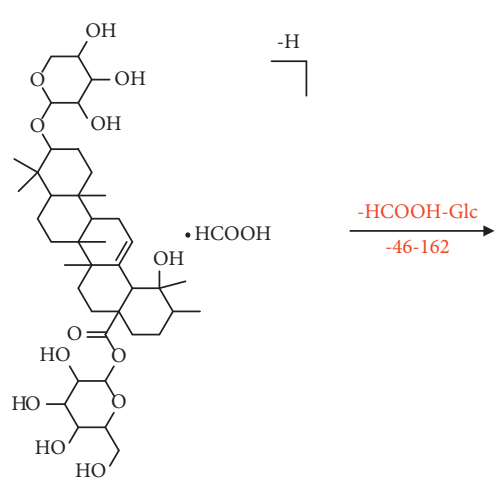

Exact Mass: 811.4435

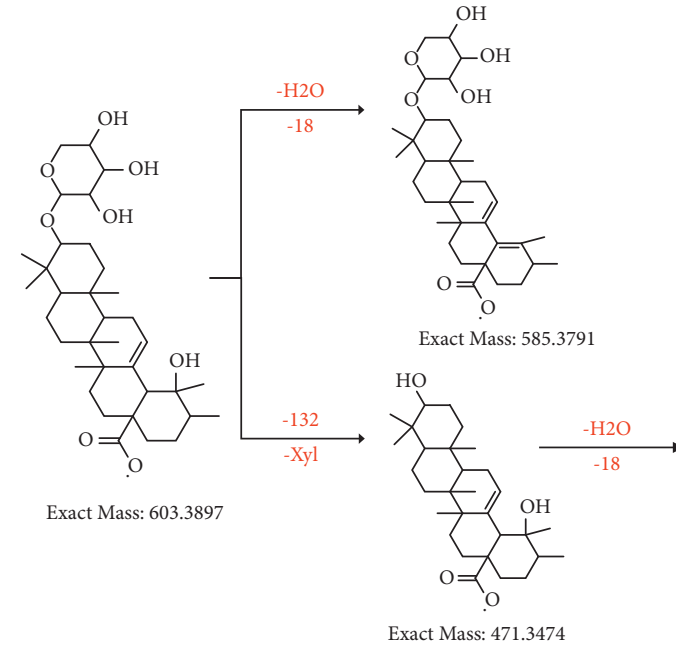

(d)

Figure 4: Continued. 
A

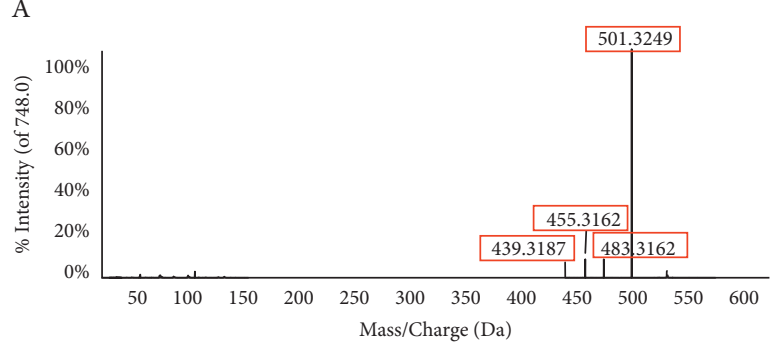

$\mathrm{C}$

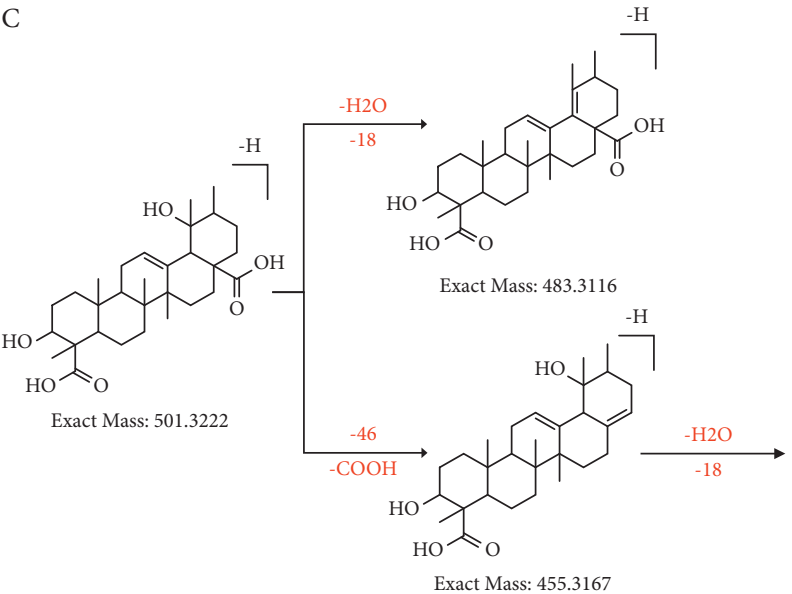

B

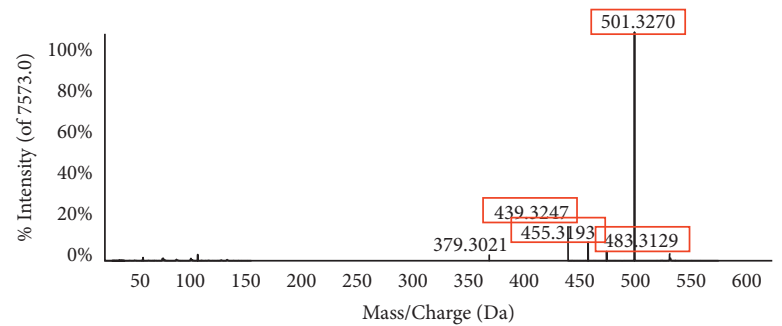

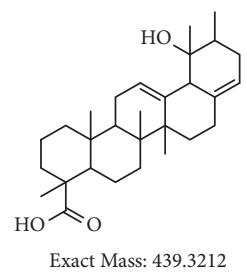

(e)

FIgURE 4: MS/MS of IRC and standard compounds. (a) Ilexsaponin A, (b) pedunculoside, (c) rotundic acid, (d) kudinoside H, and (e) ilexgenin A (A: MS/MS of IRC; B: MS/MS of standard compounds; C: mass spectrum fracture mode).

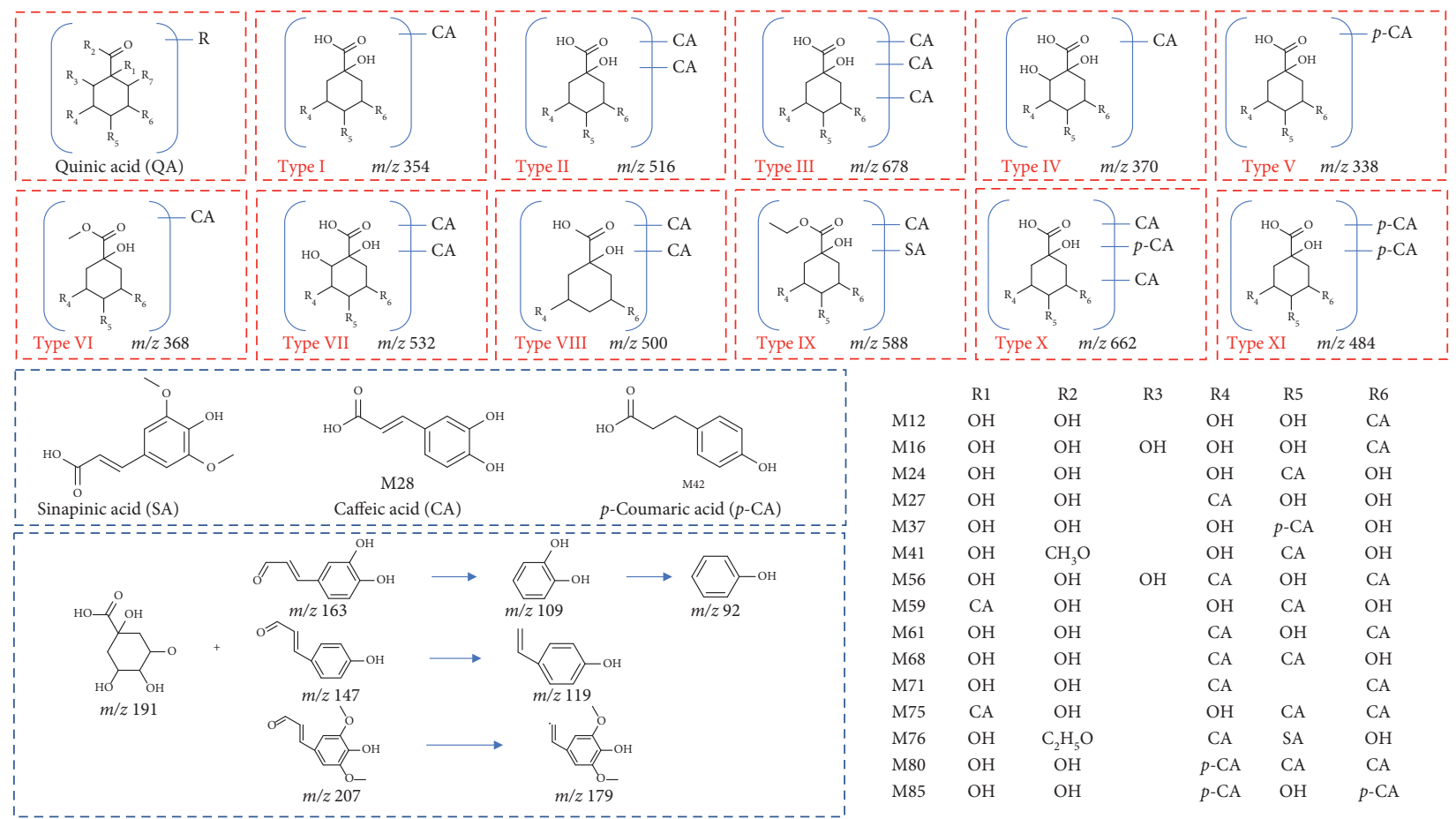

FIGURE 5: Chlorogenic acids in IRC. Quinic acid as the parent nucleus was formed mainly by introducing QA, SA, CA, and $p$-CA groups.

corresponding to $m / z 223.0612$, [CA-H] ${ }^{-}$corresponding to $\mathrm{m} / z$ 179.0350, and $[p-\mathrm{CA}-\mathrm{H}]^{-}$corresponding to $\mathrm{m} / \mathrm{z}$ 165.0557 were diagnostic ions.
To provide a basis for the identification of chlorogenic acids, six standard chlorogenic acids derivatives were used in this study, among which three were monosubstituted and 
(a)

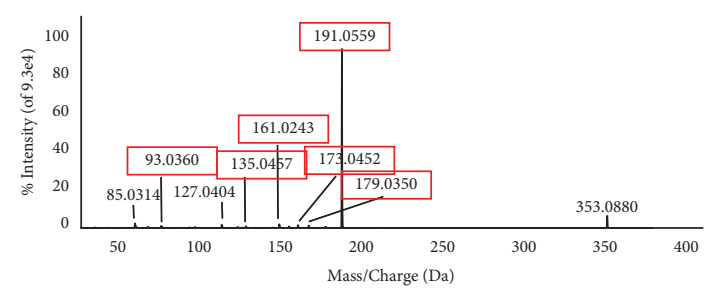

(b)

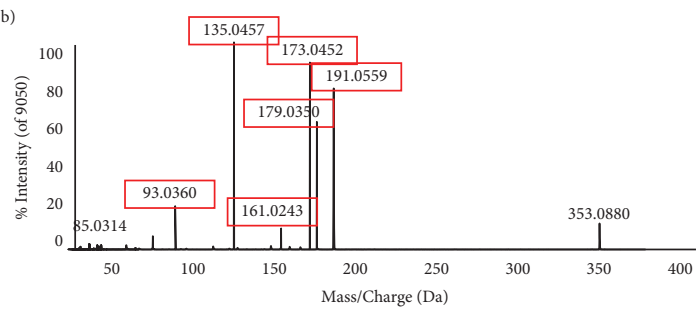

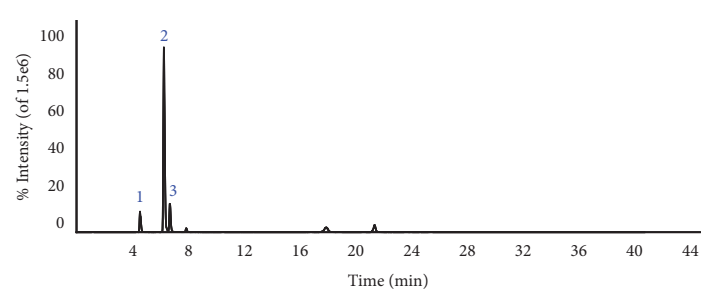

(d)

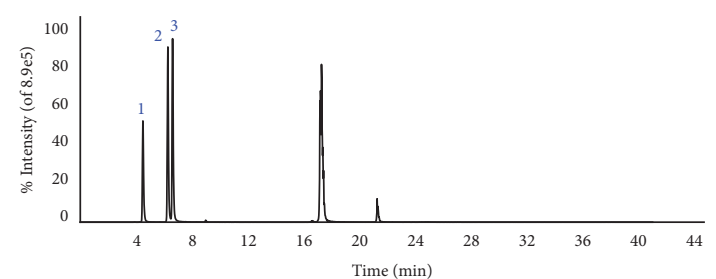

(e)

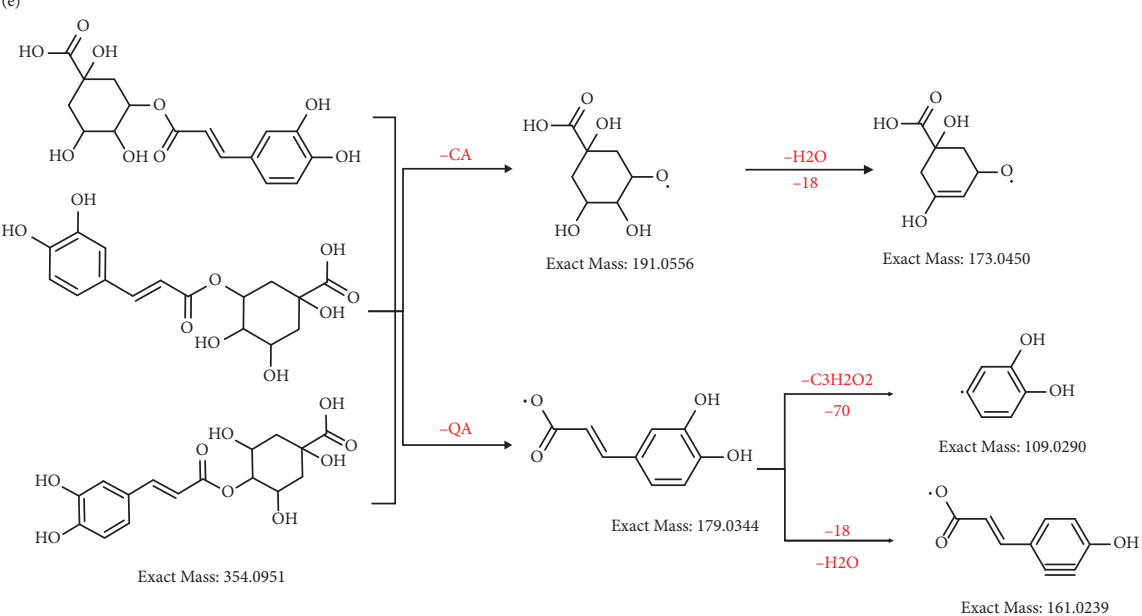

(a)

FIgURE 6: Continued. 

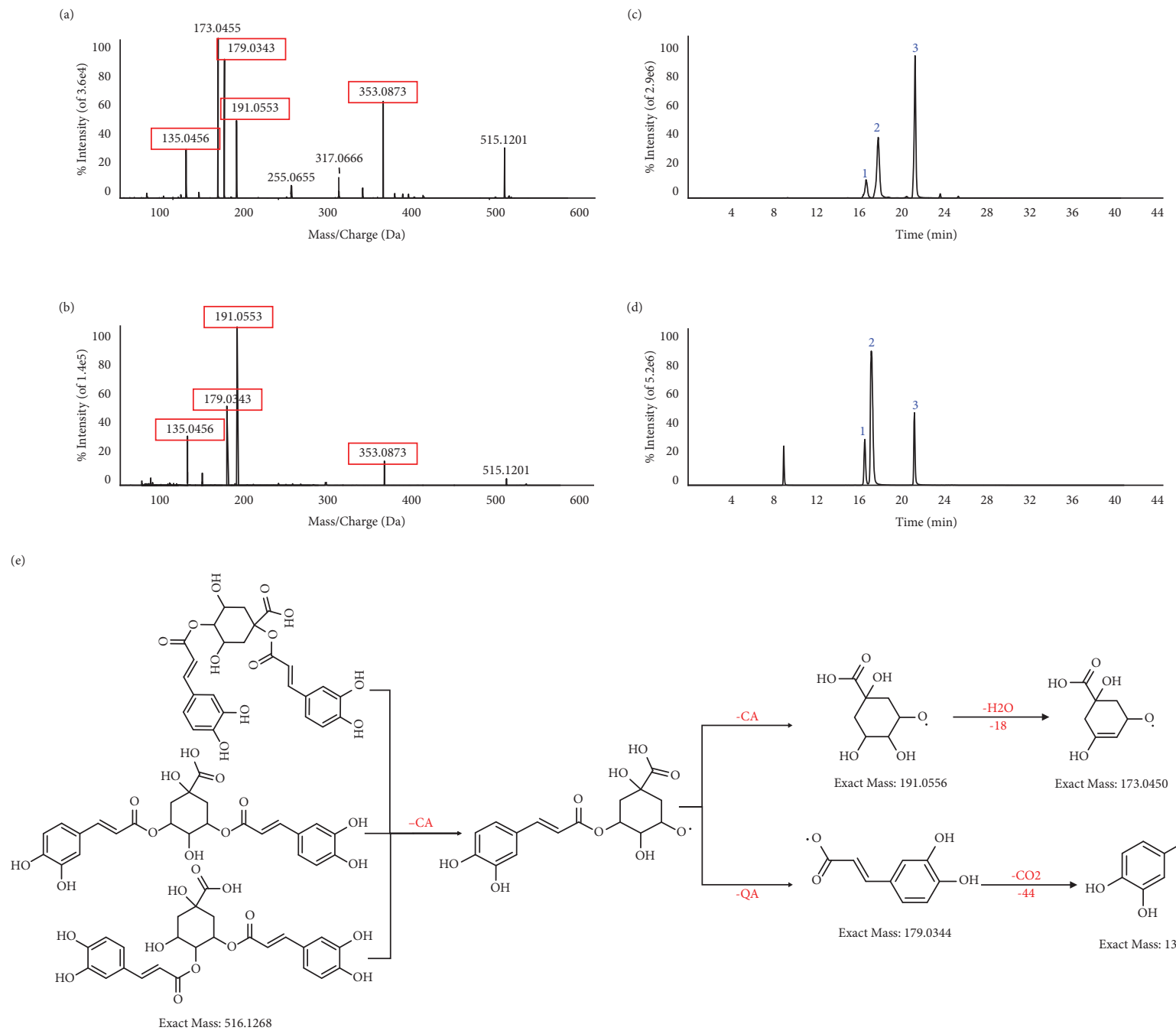

(b)

Figure 6: (a) Chlorogenic acid (1), neochlorogenic acid (2), and cryptochlorogenic acid (3). (b) 1,4-Dicaffeoylquinic acid (1), 3,4-dicaffeoylquinic acid (2), and 3,5-dicaffeoylquinic acid (3) (A: MS/MS of IRC; B: MS/MS of standard compounds; C: UV of IRC; D: UV of standard compounds; E: mass spectrum fracture mode).

three were disubstituted. Chlorogenic acid, cryptochlorogenic acid, and neochlorogenic acid are isomers, and it was challenging to distinguish even by using MS. A similar predicament was observed in the case of 1,4-dicaffeoylquinic acid, 3,4-dicaffeoylquinic acid, and 3,5-dicaffeoylquinic acid. Nonetheless, it was likely to differentiate them by comparing their peak times with the reference standards (Figure 6).

3.4. Cleavage Rules of Glycosides. A total of 33 phenylpropanes with their glycosides and 12 glycosides were confirmed in this study. All glycosides were linked mainly by Glc, Rha, Xyl, or arabinose (Arab). The diagnostic ions at $\mathrm{m} /$ $z 179.0556$ and 162.0528 corresponded to Glc. It is worth noting that $m / z 179.0556$ was different from 179.0344 of the dicaffeoyl group [26]. Moreover, the diagnostic ions at $\mathrm{m} / \mathrm{z}$ 163.0606 and 146.0579 were in accordance with Rha and $\mathrm{m} / \mathrm{z}$ 149.0450 and 132.0423 corresponded with Xyl or Arab. These monosaccharides could link with different sugar chains including Ara-Xyl, Ara-Rha, Ara-Rha-Glc, and GlcGlc. Among them, syringin (M25), belonging to phenylpropanoids, is listed as a marker in the Chinese Pharmacopoeia 2020 edition. The MS/MS of syringin was found to correspond perfectly with the reference standard. This finding provides a basis for the identification and speculation of these compounds. The representative glycoside structures of compounds are shown in Figure 7.

3.5. Cleavage Rules of Iridoid. During the determination of iridoids using MS, although extensive data have been reported, plentiful basic parent nuclei of iridoids, such as aromatic rings and aromatic heterocycles lacking regular fracture mode, were detected. Thus, iridoids could crack more chemical bonds upon bombardment with electrons. Considering the various chemical components present in IRC, the collision energy that was used in this study failed to lead to ring-opening; however, some fracture modes such as 


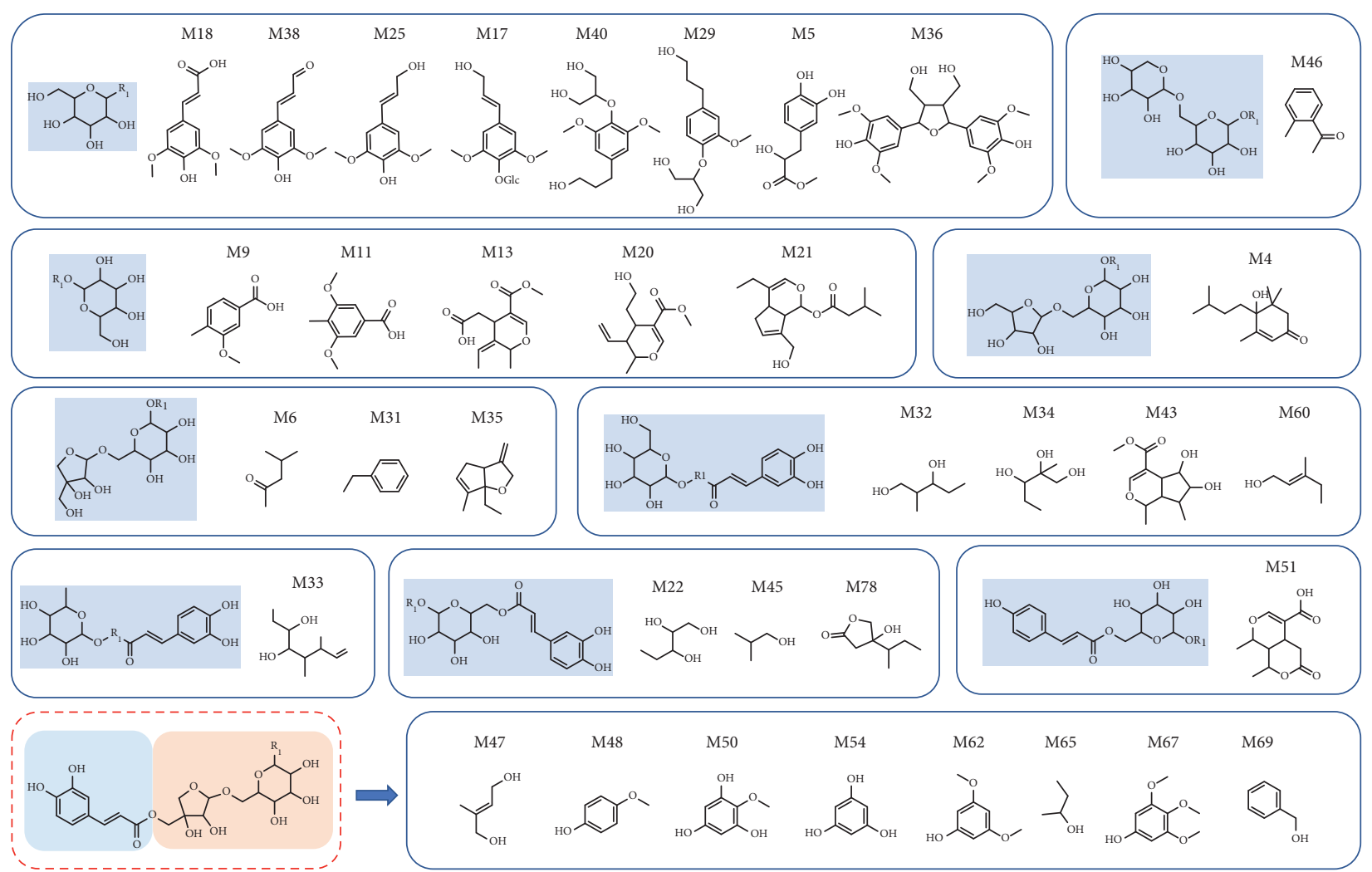

Figure 7: Representative glycoside structures. Glycosides are formed when a class of nonsugar compounds is combined with a sugar.

sugar dropping and dehydration were identified [27]. We found that M3, M4, and M64 in IRC were iridoid components of terpenoids.

3.6. Cleavage Rules of Flavonoids. For brass compounds, the core skeletons of the $\mathrm{A}$ and $\mathrm{B}$ rings were preserved, and the sum of the mass-charge ratios of fragment $\mathrm{A}$ and corresponding fragment $\mathrm{B}$ was found to be equal to the masscharge ratios of molecular ions. Thus, it was of great significance for identification in this study. For instance, M53 follows this pattern in IRC. After dropping the glycosyl moiety, the ring opened to form fragments A and $\mathrm{B}$.

3.7. Cleavage Rules of Lignans. Lignans are composed of two C6-C3 units, most of which have a circular structure. Therefore, MS data usually show a high abundance of molecular ion peaks. The structure of the benzene ring and hydrocarbyl ring in lignans is helpful in obtaining a series of molecular fragment peak information in the mass spectrum [28]. In IRC, M70, M52, and M51 were cleaved by desaccharification and the removal of dicaffeoyl group. Besides, M66 and M58 corresponded to dibenzylbutane lignan. M83 corresponded to arylnaphthalene lignan, and M55 to dibenzylbutyrolactone lignan. M49, M63, and M57 corresponded to furofurans.

\section{Conclusions}

It is well known that traditional Chinese medicine has a complicated chemical composition, which poses a challenge during analysis. However, it is important to develop effective methods to identify and utilize the constituents of traditional Chinese medicine. In this study, we report a rapid yet effective UPLC-MS/MS method, which combined a two-step data mining to screen and identify target compounds. Accordingly, 105 components (22 triterpenoid saponins, 15 chlorogenic acids, 33 phenylpropanoids and phenylpropanosides, 3 iridoids, 1 flavonoid, 10 lignans, 12 glycosides, and 9 other compounds) from IRC were rapidly identified. In combination with neutral loss, diagnostic ion, and characteristic ion filtration, the composition of IRC was analyzed on the basis of MS for the first time. Our findings show that UPLC-MS/MS could be an important means for the identification and discovery of pharmaceutical components in complex medicinal preparations. Our method is reasonable, effective, and feasible and can be used for the analysis of complex pharmaceutical components. Moreover, our findings can provide a theoretical basis for further studies on active substances and the quality control of IRC.

\section{Data Availability}

The data used to support the findings of this study are available from the corresponding author upon reasonable request. 


\section{Conflicts of Interest}

The authors declare no conflicts of interest.

\section{Authors' Contributions}

Xinxin Chen and Junmao Li contributed equally to this work.

\section{Acknowledgments}

The authors thank Guangxi University of Chinese Medicine and Jiangxi University of Traditional Chinese Medicine for its instrument support and the contribution of every participant. This project was supported by Guangxi Science and Technology Base and Talent Special Project (2018AD19034), the Project of Guangxi Overseas "100 persons plan" Highlevel Expert, Qihuang Project High-level Talent Team Cultivation Project of Guangxi University of Chinese Medicine (2021002), and National Natural Science Fund (81860711).

\section{References}

[1] L.-C. Zhao, Y. He, X. Deng et al., "Ultrasound-assisted extraction of syringin from the bark of Ilex rotunda thumb using response surface methodology," International Journal of Molecular Sciences, vol. 13, no. 6, pp. 7607-7616, 2012.

[2] B. Yang and H. Li, "Effects of gut microbiota and ingredientingredient interaction on the pharmacokinetic properties of rotundic acid and pedunculoside," Planta Medica, vol. 85, no. 09/10, pp. 729-737, 2019.

[3] M. A. Yucui, C. Wang, and W. Wang, "An HPLC fingerprint identification of dried barks of Ilex rotunda and Ilex godajam," Medicinal Plants, vol. 33-39, 2018.

[4] K. Amimoto, K. Yoshikawa, and S. J. C. Arihara, "Triterpenoid saponins of aquifoliaceous plants. VIII. Ilexosides XXIXXXXII from the leaves of Ilex rotunda THUNB," Chemical and Pharmaceutical Bulletin, vol. 40, pp. 3138-3141, 1992.

[5] W. J. Liu, Y. Y. Peng, and H. Chen, "Triterpenoid saponins with potential cytotoxic activities from the root bark of Ilex rotunda Thunb," Chemistry and Biodiversity, vol. 14, no. 2, 2017.

[6] X. Li, M. Kang, and N. Ma, "Identification and analysis of chemical constituents and rat serum metabolites in lycopodium clavatum using UPLC-Q-TOF/MS combined with multiple data-processing approaches," Evidence-based Complementary and Alternative Medicine, vol. 2019, Article ID 5165029, 8 pages, 2019.

[7] N. A. Feto, A. Mitema, and S. J. F. A. Chaudhary, "Development and validation of TOF/Q-TOF MS/MS, HPLC method and in vitro bio-strategy for aflatoxin mitigation," Food Additives \& Contaminants. Part A, Chemistry, Analysis, Control, Exposure \& Risk Assessment, vol. 37, no. 12, pp. 2149-2164, 2020.

[8] B. Yang, S. Xuan, Q. Ruan et al., "UPLC/Q-TOF-MS/MSbased metabolomics revealed the lipid-lowering effect of ilicis rotundae cortex on high-fat diet induced hyperlipidemia rats," Journal of Ethnopharmacology, vol. 256, Article ID 112784, 2020.

[9] B. Kim, J. W. Han, M. T. Ngo et al., "Identification of novel compounds, oleanane- and ursane-type triterpene glycosides, from Trevesia palmata: their biocontrol activity against phytopathogenic fungi," Scientific Reports, vol. 8, no. 1, 2018.

[10] N.-N. Song, L.-M. Yang, M.-J. Zhang, R.-F. An, W. Liu, and X.-F. Huang, "Triterpenoid saponins and phenylpropanoid glycoside from the roots of Ardisia crenata and their cytotoxic activities," Chinese Journal of Natural Medicines, vol. 19, no. 1, pp. 63-69, 2021.

[11] L. Yao, L. U. Jun, and J. Wang, "Advances in biosynthesis of triterpenoid saponins in medicinal plants," Chinese Journal of Natural Medicines, vol. 18, no. 6, pp. 417-424, 2020.

[12] B. Ma, S. Yang, J. Li et al., “A four-step filtering strategy based on ultra-high-performance liquid chromatography coupled to quadrupole-time-of-flight tandem mass spectrometry for comprehensive profiling the major chemical constituents of Akebiae Fructus," Rapid Communications in Mass Spectrometry, vol. 33, no. 18, pp. 1464-1474, 2019.

[13] W. Cai, K. Li, P. Xiong et al., "A systematic strategy for rapid identification of chlorogenic acids derivatives in duhaldea nervosa using UHPLC-Q-Exactive orbitrap mass spectrometry," Arabian Journal of Chemistry, vol. 13, no. 2, pp. 3751-3761, 2020.

[14] B. Alica, J. Silvia, B. Ľubomír et al., "Analysis of caffeine and chlorogenic acids content regarding the preparation method of coffee beverage," International Journal of Food Engineering, vol. 17, no. 5, pp. 403-410, 2021.

[15] D. Habtamu and A. Belay, "First order derivative spectra to determine caffeine and chlorogenic acids in defective and non-defective coffee beans," Food Sciences and Nutrition, vol. 8, no. 9, pp. 4757-4762, 2020.

[16] S. Zare, H. Mirkhani, O. Firuzi et al., "Antidiabetic and cytotoxic polyhydroxylated oleanane and ursane type triterpenoids from Salvia grossheimii," Bioorganic Chemistry, vol. 104, Article ID 104297, 2020.

[17] L. Hu, C. Zhou, Y. C. Huang et al., "HPLC coupled with electrospray ionization multi-stage tandem MS and TLC analysis of flavones-C-glycosides and bibenzyl of Dendrobium hercoglossum," Journal of Separation Science, vol. 43, no. 20, pp. 3885-3901, 2020.

[18] V. T. Nguyen, M. T. Nguyen, and N. Q. Nguyen, "Phytochemical screening, antioxidant activities, total phenolics and flavonoids content of leaves from persicaria odorata polygonaceae," Materials Science and Engineering, vol. 991, Article ID 012029, 2020.

[19] R. Jiang, Q. Gao, and D. Sui, "Use of 3,4-dihydroxy coffee acyl group tartaric acid in preparing medicaments for coronary disease," Europe PMC, 2008.

[20] V. I. Deineka, M. Y. Tretyakov, E. Y. Oleiniz et al., "Determination of anthocyanins and chlorogenic acids in fruits of aronia genus: the experience of chemosystematics," Russian Journal of Bioorganic Chemistry, vol. 46, pp. 1390-1395, 2020.

[21] J. Liu, Y. Liu, Y. Wu, Z. Dai, and S. Ma, "Rapid analysis of aristolochic acid analogues in traditional Chinese patent medicine by LC-MS/MS," Journal of Analytical Methods in Chemistry, vol. 2020, Article ID 8823596, 7 pages, 2020.

[22] A. Sharma, S. C. Sati, and O. P. Sati, "Triterpenoid saponins from the pulp of Sapindus mukorossi and their antifungal activities," Phytochemistry, vol. 20135 pages, 2012.

[23] T. Hanh, P. T. Cham, H. Do et al., "Iridoid glucosides and phenylethanoid glycosides from Plantago major," Phytochemistry Letters, vol. 39, pp. 111-115, 2020.

[24] S. Jeong, J. Jang, H. Cho, and Y.-B. Lee, "Simultaneous determination of three iridoid glycosides of Rehmannia glutinosa in rat biological samples using a validated hydrophilic interaction-ultrahigh performance liquid chromatography- 
MS/MS in pharmacokinetic and in vitro studies," Journal of Separation Science, vol. 43, no. 22, pp. 4148-4161, 2020.

[25] D. L. Ma, Y. Luo, R. Huang et al., "Cornel iridoid glycoside suppresses hyperactivity phenotype in $\mathrm{rTg} 4510$ mice through reducing tau pathology and improving synaptic dysfunction," Current Medical Science, vol. 40, no. 6, pp. 1031-1039, 2020.

[26] X. Xu, W. Wang, Z. Zhao et al., "Effects of total iridoid glycosides of Picrorhiza scrophulariiflora against non-alcoholic steatohepatitis rats induced by high-fat and high-sugar diet through regulation of lipid metabolism," Chinese Herbal Medicines, vol. 12, no. 1, pp. 67-72, 2020.

[27] H. Jin, G. Tang, J. Li, L. Ma, Y. Li, and Y.-X. Chang, "Simultaneous determination of phenolic acids, anthraquinones, flavonoids, and triterpenes of cynomorii herba in different harvest times by LC-MS/MS," Journal of Analytical Methods in Chemistry, vol. 2020, Article ID 8861765, 9 pages, 2020.

[28] X. L. Cheng, F. Wei, J. Chen et al., "Using the doubly charged selected ion coupled with MS/MS fragments monitoring (DCSI-MS/MS) mode for the identification of gelatin species," Journal of analytical methods in chemistry, vol. 2014, Article ID 764397, 7 pages, 2014. 